No harm has been done, and many have improved, but the writer is not satisfied that the treatment is here of any decided value, and believes that simple rest and the resisted movements are much to be preferred.

Cases in which it is contraindicated.-These are aneurysm, marked arteriosclerosis, chronic myocarditis and chronic Bright's disease, yet, while baths are in these cases distinctly dangerous and harmful, the movements may be decidedly beneficial.

Precautions.-To obtain the best results, the tub should be large and the patient immersed to the chin if possible. The baths should never be given hot, nor, on the other hand, should the patient feel any chill during the bath. Great pains must be taken to avoid voluntary movements during and after the bath. If the circulation in the extremities be poor, brisk, light friction may be used. If calcium chlorid be used it must be pure. A very sick patient must invariably be watched by a skilled attendant throughout the bath. If $\mathrm{CO}_{2}$ is actively generated it is well to fan it away before placing the patient in the bath. Cases of extreme incompensation are best treated at the outset by rest in bed, and the usual remedies; they will almost always show marked improvement, and when this improvement begins to flag one may begin the baths and use the exercise with slight daily increase in the resistance. Allow an exceedingly gradual resumption of voluntary movement and accept no case of severe incompensation for less than six weeks of treatment.

The following rules must be observed in using the movements: Remember that they are intended to produce the beneficial result of exercise without fatigue, and you have the keynote of success, and can appreciate the scrupulous care necessary in using them in chronic heart cases. The nicest judgment and most painstaking skill are required, and increase of the pulse-rate, pallor, or evidence of dyspnea warns us to stop at once. The amount of resistance, rest intervals, and number of movements well borne by the patient must be left to the operator's good sense. Furthermore, the clothing must be loose; each movement should be performed deliberately and smoothly; the breathing must be deliberate and regular; the resistance should be varied according to the strength of the patient,and the effect produced; and no move. ment should be repeated in the same limb or muscle group. Thus simple and rational are the principles of this treatment.

Results obtained.-If proper care in the selection of cases and strict supervision be exercised, the results are really remarkable. The writer has never seen such extraordinary shrinkage during the first bath as has been described by some writers ( 3 inches), but in an old mitral case a man must be strongly biased or a trifle weak in his physical diagnosis if he can not easily demonstrate a decided recession of the borders during the bath. That this is not due simply to the somewhat deeper breathing induced by the bath, as has been asserted, may be easily demonstrated by testing the effect of similar breathing before the bath. It must be remembered, however, that rest alone may produce remarkable shrinkage in a dilated heart, and it is probably wise to try rest, digitalis and strychnin for the first week or two and thus get a very gradual rather than rapid change in the conditions. As before stated, we always arrive at a point where progress is slow, and then the baths are indicated. While the writer believes that the effect of the baths is often overestimated, by reason of the fact that the effect of simple rest is underestimated, jet the shrinkage of the cardiac area, the improvement of the heart action, the pronounced diuresis, and the prompt removal of secondary symptoms under this rational and simple treatment are decidedly impressive and, in the writer's opinion, no treatment of dilated heart can leave the salt baths and movements out of that great therapeutic combination which includes digitalis and strych. nin, baths and resisted movements, walking on the flat and hill-climbing. The rational therapeutist can omit none of these from his list, yet he might find it hard to fix their relative importance. If the writer were forced to discard all means but one, baths and resisted movements would be his choice as being of the broadest application. But fortunately we are not thus limited, and the measure under discussion forms in any event one of the most important adjuncts to the successful treatment of heart disease.

\section{NOTES ON TYPHOID FEVER.}

TWO HUNDRED AND TWENTY-FIVE CASES TREATED DURING AN EPIDEMIC OF THAT DISEASE.

Presented to the Section on Materia Medica, Pharmacy and Therapeutics at the Forty-ninth Annual Meeting of the American Medical Association held at Denver, Colo., June 7-10, 1898. BY J. P. BARBER, M.D.

$$
\text { MINEAPOLIS. }
$$

Typhoid fever remains a most interesting study. Its pathology, which was supposed to have been settled years ago by the discovery of the bacillus of Eberth, will have to be remodeled to fit certain facts of recent research. The bacteriologist, who has done so much to illuminate this disease, can not yet call his labors finished. The best efforts of the therapist to evolve a treatment which will abort, or even materially modify, the course of the disease have signally failed. The clinician must often confess his shortcomings in its diagnosis.

An epidemic of this disease began in the city of Minneapolis in February, 1897, and was traceable directly to the water-supply. Drs. Westbrook and Wilson, ${ }^{1}$ of the laboratory of the State Board of Health, isolated the bacillus typhosus from the water in April of that year. Nearly every case seen during the months of March, April and May was typic and severe. The spring floods, which came about the middle of May, were unusually severe and the river, from which the water-supply is taken, remained comparatively high during the remainder of the summer and fall. Then followed a series of most interesting cases, more than half of which were atypic. The total number of cases seen by the writer from March 1, 1897 , to May 1, 1898, was 225 . Of this number 114 were typic and could have been easily diagnosticated clinically. The remaining 111 were atypic, and many of them could not have been identified without the aid of certain laboratory tests. For convenience these 111 cases are divided into three classes, as follow: 1, the extremely mild, ó cases; 2, abortive, 28 cases; 3. beginning concurrently with some other disease, 28 cases.

The first class of cases is well illustrated by the fol. lowing history:

Class 1.-C. B., male, aged 32, had been feeling ill three days; headache, anorexia, lumbar pains, general muscular soreness; temperature 99 degrees $\mathrm{F}$. at 3 P.M. ; tongue slightly furred 
with white coating; slight tenderness on deep pressure over right iliac region; diazo-reaction and serum test both negative.

Fourth day. Temperature, 9 A.M., 97.6 ; 3 P.M., 99 degrees ; other symptoms about the same; urine and blood tests both negative.

Fifth day. Temperature, 9 A.M., 97.8, and 5 P.M., 99 degrees ; headache and muscular pains increased ; tongue more deeply coated; bowels constipated; urine shows faint rose color with diazo-reaction; serum test negative.

Sixth day. Temperature, 9 A.M., 99, 4 P.M., 99.8 degrees. Distinct rose-colored diazo-reaction; serum test negative.

Faint grouping was coted with the serum test on the eighth day, and a perfect reaction on the tenth, twelfth, fourteenth and twentieth days. Slight enlargement of the spleen was noted on the tenth day. The temperature vacillated between 97.6 and 99 degrees from the eighth to the eleventh days, after which it remained normal. The tongue cleared off, appetite returned and the patient felt as well as usual.

This class, of which the above is a good representa. tive, presents many variations in the symptoms. But, as a rule, the usual prodromata are present, with a very low fever of ten days' to two weeks' duration. All have reacted to the diagnostic tests for typhoid fever.

Class 2.-Alice W., female, aged 13 years. Had eaten inordinately of sweetmeats three days ago; has been vomiting ever since ; intense headache, epigastric pain and tenderness. Temperature 103 , pulse 90 ; respiration 20 ; diazo-reaction and serum test absent.

Fourth day: 'Temperature, 3 P.м. 104, respiration 20 ; pulse 96 ; vomiting ceased ; tenderness over entire abdomen; diazoreaction intense; serum test negative.

Fifth day: Temperature 9 A.M. 102, 4 P.M. 103; headache not so severe; feels much more comfortable; no pain in epigastric region, but still tenderness on pressure over abdomen; area of splenic dullness considerably increased ; no spots ; serum reaction present.

Seventh day: Temperature 9 A. м. $97.5 ; 4$ P.м. 99. She feels well and wants to sit up. The temperature registered between 97.5 and 99 for three or four days, the tongue cleaned off, appetite returned and she felt as well as usual. The serum reaction was present on the 6 th, 8 th, 9 th, 12 th and 20 th days.

This is a fair representative of this class of cases. The outset is generally rapid, sometimes sudden with chill or, if the patient be a child, convulsion. The temperature rises to 104 or 105 , or even higher, for three or four days, then suddenly the disease terminates by crisis similar to that of pneumonia. There is generally no cough in these cases, and in none of them could there be detected the slightest physical sign of lung involvement. None of them have shown the rose-colored spots. In some, splenic enlargement could be plainly detected. There was sometimes tenderness in the epigastric, or in the right iliac region, but often these signs were wanting. All of them reacted to the urine and blood tests, and as a rule each test appeared early in the disease, the diazoreaction almost invariably appearing first.

Class 3.-Pneumonia, 7 cases, severe acute bronchitis 10 , amygdalitis 3 , otititis media 1 , pleuritis 2 , peritonitis 1 , renal hematuria 2 , cystitis 1 , periostitis 1 .

I would call attention to this as a very interesting class of cases. In 23 of them there is a suspicion that the respiratory tract was the point of invasion of the infection. Sicard ${ }^{1}$ believes that one-tenth of all cases are infected in this manner. Henrots' ${ }^{2}$ report of 112 cases of typhoid fever among a troop of 1600 soldiers, who during a sham battle passed over a very dry and dusty field which had been fertilized with human excrement, would indicate the respiratory tract as a point of invasion of the bacillus typhosus, especially as it was noted that a great number of them had pneumonia and other pulmonary complications. Flexner's ${ }^{3}$ case, in which the typhoid bacillus was found in the lung, liver and spleen, while the intestinal mucous membrane was normal, and the mesenteric glands not enlarged, would seem to add additional weight to this theory. There is abundant evidence in the recent literature of this disease to show that the intestinal lesions are not a necessary part of its pathology. Nichols and Keenan ${ }^{4}$ have recently reported a case and collected nine others in which no lesion of the intestine was found, although the typhoid bacillus was cultivated, usually from the spleen. Sanarelli ${ }^{5}$ maintains that the disease is, properly speaking, not an intestinal one; that the spleen and lym. phatic system are the points where the bacilli produce their peculiar toxins, and the intestinal lesions are due to secondary causes. Osler ${ }^{6}$ makes the clinical observation that "the severity of the symptoms of typhoid fever bears no relation to the extent or intensity of the intestinal lesions."

There have been two deaths; a little less than 1 per cent. This is not mentioned to bolster up any particular treatment, but rather, to show the fallacy of mere figures, unconnected with other facts, when used to prove the efficacy of any so-called abortive treatment. One-third of these cases have either aborted before the tenth day or run a course so mild as to make the diagnosis of scientific interest without the use of the particular remedies which are claimed to produce these results. While not decrying medicinal treatment in this disease, the writer believes that other factors play a much greater part in affecting the mortality. The attenuation of the specific infection, the amount received, the individual resistance or degree of immunity, may be mentioned among these factors; and the peculiar circumstance of this epidemic would tend to show that at least one of them had much to do with lessening its severity.

It is desirable to call attention to the value of the chemic and bacteriologic tests in the diagnosis of this disease. In a former article the writer has shown the relative values of these two tests. The diazo-reaction appeared first or at the same time in 80 per cent. of the cases in which it was used side by side with the serum test. It has failed to appear in 12 out of 200 cases, and in only one of these was it fairly tried. It almost always appeared before the eighth day, even in the mildest cases; but in some of these it is present only on one or two days. To be certain that it has not appeared,tests should be made daily. Although it is found in some other diseases - measles, scarlatina, parotiditis, varicella, tuberculosis and cancer-by careful attention to the clinical symptoms, this test is a valuable aid in making an early diagnosis in typhoid fever. Simon's ${ }^{8}$ method, with Green's ${ }^{9}$ modification of the formula, has been used and no test accepted unless a pink or rose color was given in dilution with five to ten parts of water.

The serum test is the anchor in the diagnosis of this disease, and is very nearly, if not quite, pathognomonic. Dr. Wilson, ${ }^{10}$ assistant bacteriologist to the Minnesota State Board of Health, has made 1450

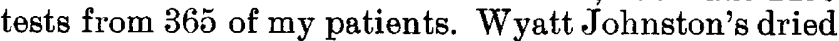
blood method with dilution of about 1-25 was used in about one-half the tests, and Dr. Wilson's ${ }^{10}$ method of collecting the blood on aluminum foil, weighing and diluting it accurately to 1-25, was used in the latter half. Of those cases in which the reaction was negative throughout, one was typic typhoid fever clinically. Two others were twin infants ten weeks old whose temperature ranged from 100 to 103 for three weeks. Each had rash which was not typic. 
They had diarrhea, distension of the bowels and enlarged spleen. The urine of each gave diazo-reaction throughout the illness. They had been partly bottle-fed, the family using infected water. No other members of the family had typhoid fever. One other case of doubtful diagnosis was reported by the writer ${ }^{11}$ in a former article. These four would make a percentage of about 1.8 in which the reaction was absent. On the other side there were two cases, one of which has been reported, ${ }^{12}$ in which the reaction was present during the course of another disease, in which there was at least a suspicion that typhoid fever was not present. The second case was one of severe erysipelas, during the course of which the serum reaction appeared on the fifteentil day. The spleen was enlarged, and there was diarrhea with characteristic stools. There was at the same time another case of typhoid fever in the family.

Gwyn's ${ }^{13}$ case of typic clinical typhoid fever with rose spots, diazo-reaction, hemorrhage from the bowels, serum reaction negative, in which cultures from the blood showed only Gaertner's bacillus, may throw some light on such reports as Brill's, ${ }^{14}$ who, in seventeen cases clinically resembling typhoid fever found the serum test negative. And further research along this line may prove Widal's test pathognomonic of infection with the bacillus typhosus.

BIBLIOGRAPHY.

1 Wilson and Westbrook: British Medical Journal, Dec. 18, 1897. 2 London Lancet, Feb. 1, 1896.

3 Flexner: Johns Hopkins Bulletin, December, $189 \%$

Nichols and Keenan: Montreal Medical Journal, January, 1898

Sanarelli: Ann de linstitut Pasteur, April, 1894.

Osler : Philadelphia Medical Jourti, Jan. 1, 1898

\& New York Medical Journal, A pril 16, 1808 .

8 Simon: Clinical Diagnosi

Medical Association, Feb, 4,1894

o Westbrook and Wilson: Philadelphia Medical Journal, March 26, 1898 . 12 New York

3 Gwyn: Johns Hopkins Hospital Bulletin, March, 1898

14 Brill: New York Medical Journal, Jan. 8, 1898.

\section{CLIMATIC VERSUS SERUM TREATMENT OF PULMONARY TUBERCULOSIS.}

Presented to the Section on Materia Medica, Pharmacy and Therapeupeutics, at the Forty-ninth Annual Meeting of the American Medical Association, held at Denver, Colo., June 7-10, 1898.

BY F. E. WAXHAM, M.D.

PROFESSOR OF MEDIOINE AND XARYNGOLOGY, COLORADO SCHOOL OF MEDICINE.

DENVER, COL.

The climatic treatment of disease is a most impor. tant subject, and one that I am convinced has not been fully studied and appreciated. No doubt many failures are to be attributed to the fact that neither proper cases nor proper climate have been selected. Because a patient has tuberculosis is no reason or excuse why he should be sent two thousand miles or more from home and comfort and friends, only to find, perhaps, his malady increased, and to die among strangers. We should prescribe a change of climate with as much discretion and judgment as any other remedy. Whether a patient should be sent from come in search of health will depend upon several conditions. The stage of the disease, the vigor, vitality, disposition of the patient, and the means with which to live comfortably, are all to be considered. Physicians in the Rocky Mountain region are continually meeting with cases that should never have been sent to this country. Too frequently we are obliged to advise their immediate return. Tuberculosis in the last stages is only aggravated, and the condition of the patient made worse and more uncomfortable by a residence in this altitude.
Climatic treatment, like any other treatment of disease, should be applied early. The earlier the application of the remedy, the more certain and positive the results. It may be eaid, with little fear of contradiction, that tuberculosis in the first stage, and often in the secund, is cured by a proper change of climate. In selecting cases for climatic treatment, therefore, by all means select the early ones. Do not wait until the golden opportunity is lost in the hope. of arresting the disease by less effective measures.

It must be stated in this connection, however, and. it must be admitted, that tuberculosis originating in this climate, as a rule, runs a rapid and unfavorable. course. This has been the observation of those who have had the opportunity of watching these cases. It has been my misfortune to have met with a few such patients, and they have formed no exception to. the rule.

There is another factor of no little importance that should be considered in the selection of cases appropriate for climatic treatment, and that is, the financial circumstances of life. Many seem to entertain the impression that no matter what the stage of the disease, if they can only live to reach this land of promise, their ills will take wings; they will find ready employment and health will soon be restored. By the irony of fate, many find no employment, and, as a result of anxiety and forced economy, the ills are increased. If a patient's circumstances are such that he can not live in comfort and idleness, if necessary, for at least a year, it would seem hopeless to send him in search of health. Something more is needed than mere climate. A contented mind, freedom from worry and anxiety, good and abundant food and clieerful surroundings - these are all essential factors. I contend that it is more humane to allow a patient to die in peace at home, even if his days are shorter, than to send him in the later stages away in search of health, unless he has means with which to command every comfort.

The selection of the proper climate is quite as important as the proper selection of cases. There is no climate suitable to all cases, although in general terms we may say that the essential features are altitude, dryness, abundant sunshine, and uniformity of temperature. Wherever these conditions prevail, a tubercular patient has the best chance to recover. Early cases, with no heart lesions do best, as a rule, in a high altitude with its invigorating and stimulating atmosphere. These are the cases that do re. markably well in Colorado. More advanced cases need a warmer and a less elevated climate. Such cases do better in New Mexico, Southwestern Texas, Arizona, or the interior of Southern California. Many cases do well in Colorado during the summer and fall, but do not bear well the cold of winter, and it becomes necessary for them to go southward during this season; and, again, there are many who do wonderfully well in winter in New Mexico, Texas or Arizona, but who can not endure the heat of summer in those regions. It becomes necessary, therefore, to shift a patient about from one climate to another in order to take advantage of the best seasons until he has recovered.

Another important consideration is the fact that tubercular patients recover slowly. It requires from six months to three or four years, according to the condition of the patient and the stage of the disease, to entirely recover, and even after this period it is 